Conclusions SC complicated by psychiatric symptoms may be difficult to manage as both physical and psychiatric symptoms require on-going collaborative assessment and treatment within paediatric services and CAMHS.

\section{G57(P) TO PRESENT A CASE OF PERINATAL ARTERIAL ISCHAEMIC STROKE (PAIS) THAT PRESENTED WITH RECURRENT FOCAL SEIZURES AT BIRTH}

A Sharma, D Jayachandran, D Agrawal. Paediatrics, Darlington Memorial Hospital, Darlington, UK

\subsection{6/archdischild-2020-rcpch.43}

Case A term baby with no antenatal risk factors was born by emergency caesarean section due to unexplained fetal tachycardia. The pregnancy was uneventful and no resuscitation was required. The baby developed right sided focal seizures at 2 hours of birth (lasted for approximately 2 minutes). Subsequently, she had multiple clusters and prolonged seizures between 2 and 24 hours of birth. The seizures continued after a maximum loading dose of Phenobarbitone $(40 \mathrm{mg} / \mathrm{kg} / \mathrm{dose})$ and so was given a loading dose of Levetiracetam at $20 \mathrm{mg} /$ $\mathrm{kg} /$ dose with good effect. Magnetic Resonance Imaging of the head was performed at 23 hours and the diffusion weighted imaging demonstrated diffusion restriction of left parieto- occipital lobe with corresponding drop on apparent diffusion coefficient mapping in keeping with left cerebral acute infarct. Haematological workup showed Low Protein C and Low Free Protein S which would be repeated at 6 months.

Discussion PAIS are cerebrovascular events secondary to arteriopathic, cardiac and haematological pathology, occurring between 20 weeks of fetal life through to the 28th postnatal day. Most commonly, this involves the middle cerebral artery territory. MR with Diffusion Weighted imaging is the radiologic investigation of choice. Maternal conditions that have been associated with perinatal stroke in the fetus include prothrombotic disorders, cocaine abuse, placental complications and placental vasculopathy. In many cases, the placenta is suspected to be the underlying embolic source for perinatal stroke, although data on placental pathology is often lacking. During the delivery process, an infant may develop a cervical arterial dissection that leads to stroke. Several conditions in the neonatal period predispose to perinatal stroke including prothrombotic disorders, congenital heart disease, meningitis and systemic infection. The outcome is variable and depends on severity, anatomic localization, and other factors. Intravenous Levetiracetam is effective in controlling seizures and could be used as a second line anticonvulsant.

Conclusion Arterial ischemic infarction occurring around the time of birth is an increasingly recognized cause of neurological disability in children and should therefore be considered as an important differential diagnosis in newborns presenting with focal neonatal seizures.

\section{G58(P) DEFINING CEREBRAL PALSY- CONSENSUS OR CONTROVERSY?}

T Condon, A Boyd Crotty, R Brennan, SR George, E Gray, A O'Donnell, N O'Sullivan, J Olaniyi, J Allen, N Lagan, J Meehan, EJ Molloy. Discipline of Paediatrics, Trinity College Dublin, Dublin, Ireland

10.1136/archdischild-2020-rcpch.44
Introduction Cerebral Palsy (CP) is a well-recognised neurodevelopmental disorder beginning in early childhood and persisting through life. CP registries from developed countries suggest its prevalence is $2-3$ per 1000 live births. It has consistently proven a challenge to define $\mathrm{CP}$, as documented by the numerous attempts. This review aimed to investigate both the consensus and controversy surrounding the definition of $\mathrm{CP}$ and to investigate which definition is currently the mostly widely accepted and applied.

Methods A literature search was conducted using EMBASE, CINAHL and SCOPUS. The search was limited to articles available in English and peer-reviewed journals. Articles were screened in four stages by two independent reviewers and the Preferred Reporting Items for Systematic Reviews (PRISMA) was used.

Results 375 articles were identified, and 41 full-text articles were included in the study. The literature outlined numerous definitions of CP. Most recently, a new consensus definition was proposed by Rosenbaum et al. which emerged from an international workshop on the definition and classification of CP, held in Maryland, July 2004. However, while widely accepted, this definition still faces criticism.

Conclusions Overall, there has been a marked evolution from the first definition of CP by Little to the current consensus definition of Rosenbaum et al. Use of epidemiological data collected by $\mathrm{CP}$ registers and modern diagnostic techniques has paved the way for adjustments of previously scrutinised definitions. However, there is a need for a worldwide consensus to maintain a high accuracy rate of diagnosis and management.

\section{G59(P) THE ROLE OF FLUORODEOXYGLUCOSE POSITRON EMISSION TOMOGRAPHY FDG-PET SCAN IN THE DIAGNOSIS OF AUTOIMMUNE ENCEPHALITIS}

AJ Jalal, AJ Jollands. Paediatrics, Ninewells Hospital, Dundee, UK

\subsection{6/archdischild-2020-rcpch.45}

The diagnosis of autoimmune encephalitis (AE) is based on clinical features and associated findings on MRI, EEG, CSF examination and neuronal antibody testing. Diagnosis may be difficult when presenting features are atypical and special investigations normal. Additionally neuronal antibody testing is not routinely available which may delay treatment. Fluorodeoxyglucose positron emission tomography scan (FDG-PET) may be an adjunct to aid the diagnosis of early disease and monitor the response to treatment. This literature review aims to establish if there are characteristic FDG-PET features associated with $\mathrm{AE}$ and/or specific patterns associated with neuronal antibody types in the first instance and secondly determine if serial FDG-PET can be used to measure response to treatment.

A total of 217 adults and children with suspected or definite AE were identified in the literature. Fifty patients had normal MRI findings but abnormal FDG-PET uptake patterns at diagnosis. Additionally 10 patients with seronegative AE had abnormal diagnostic FDG-PET findings. Serial FDG-PET data were available In 37 patients. Thirteen patients showed improvement and an additional 12 showed complete resolution after treatment which correlated with clinical improvement. There were no specific FDG-PET uptake patterns associated with acute, semi-acute or late phases of the disease however basal ganglia involvement was more commonly seen in those with ion channels and other cell surface protein 